\title{
Lidil
}

Revue de linguistique et de didactique des langues

46 | 2012

Typologie et description linguistiques

\section{Évolution du L simple intervocalique latin dans le parler occitan alpin d'Usseaux (Province de Turin, Italie)}

Lucie Amaro

\section{OpenEdition}

\section{Journals}

Édition électronique

URL : http://journals.openedition.org/lidil/3252

DOI : 10.4000/lidil.3252

ISSN : 1960-6052

Éditeur

UGA Éditions/Université Grenoble Alpes

\section{Édition imprimée}

Date de publication : 15 décembre 2012

Pagination : 175-189

ISBN : 978-2-84310-236-3

ISSN : $1146-6480$

Référence électronique

Lucie Amaro, «Évolution du L simple intervocalique latin dans le parler occitan alpin d'Usseaux (Province de Turin, Italie) », Lidil [En ligne], 46 | 2012, mis en ligne le 15 juin 2014, consulté le 01 mai 2019. URL : http://journals.openedition.org/lidil/3252 ; DOI : 10.4000/lidil.3252 


\title{
Évolution du L simple intervocalique latin dans le parler occitan alpin d'Usseaux (Province de Turin, Italie)
}

\author{
Lucie Amaro*
}

\begin{abstract}
RÉSUMÉ
Si les parlers occitans actuels du Briançonnais historique connaissent une unité au niveau lexical et morphosyntaxique, ils présentent une certaine variation et différents stades d'évolution au niveau phonologique et phonétique. Au sein de ces parlers, ceux du Val Cluson, dans la province de Turin, en Italie, présentent la particularité d'être situés à la périphérie de l'espace occitan, et à l'est du continuum des langues romanes, à la jonction entre le gallo-romain et le gallo-italique. Ces particularités font de cette variété d'occitan un sujet intéressant pour l'étude de l'apport de la typologie dans la description d'une langue.

Nos recherches se sont portées sur le village d'Usseaux (Haut-Cluson) où nous avons constaté un phénomène phonologique intéressant : l'absence de L intervocalique latin. Ce phénomène n'est pas attendu en occitan standard (languedocien), ni dans les autres langues romanes voisines, sauf en portugais, et dans quelques dialectes d'Italie septentrionale comme le vénitien. Bien que le cadre de la recherche soit synchronique, il est parfois indispensable d'avoir recours à la diachronie pour expliquer, comme ici, des phénomènes atypiques et caractéristiques d'une variété linguistique. L'analyse exploite des données de première main recueillies au cours de plusieurs missions sur le terrain et auprès de deux locuteurs natifs du village d'Usseaux.
\end{abstract}

\section{ABSTRACT}

The Occitan dialects of the alpine region are known for their relative lexical and morphosyntactical unity, but they exhibit some variation and attest different steps of evolution on the phonological and

* Lucie Amaro est doctorante au sein du Laboratoire DDL (UMR 5596, Université Lyon 2) et enseigne comme PRAG de Linguistique anglaise à l'Université Lyon 1. 
phonetic levels. Among those dialects, those of the Chisone Valley, in the province of Turin, are located at the periphery of the Occitan area, and east of the continuum of Romance languages, at the junction between Gallo-Roman and Gallo-Italic languages. These aspects make the region an interesting subject for the study of the contribution of typology to the description of languages.

Our study was conducted in a village of the Chisone Valley, Usseaux. We noticed there an interesting phonological phenomenon, that of the absence of Latin intervocalic L. This phenomenon is not expected in standard Occitan, nor in the other Romance languages, except in Portuguese and in some northern Italian dialects like Venitian. Even though the frame of our study is synchronic, it is sometimes necessary to resort to diachrony to highlight a phenomenon, or the absence of a phenomenon, as compared to related or unrelated languages. Throughout the analysis, we will be using first-hand data, collected during several field trips and coming from two native speakers from the village.

Le domaine linguistique occitan est situé au cœur de la Romania et s'étend sur trois pays : le tiers sud de la France pour sa plus grande partie (à l'exception des zones basques et catalanes au sud-ouest), dans quatorze vallées italiennes, ainsi que dans le Val d'Aran en Espagne. L'espace linguistique occitan est traditionnellement divisé en deux grands ensembles : le nord occitan et le sud occitan, qui se subdivisent eux-mêmes en sous-ensembles dont les parlers présentent une relative unité morphologique ${ }^{1}$, mais qui varient notamment en ce qui concerne la phonologie et la prosodie : gascon, languedocien et provençal (au sud) et limousin, auvergnat et vivaro-alpin, (au nord). La commune d'Usseaux est située en périphérie de ce dernier espace, au nord-ouest de l'Italie, dans le Val Cluson (carte 1).

L'étude que nous allons présenter ici s'inscrit dans un travail de doctorat en cours sur la description morphosyntaxique de la variété d'occitan alpin parlée dans la commune d'Usseaux. Bien que le cadre de la recherche soit synchronique, il est parfois indispensable d'avoir recours à la diachronie pour expliquer, comme ici, des phénomènes atypiques et caractéristiques d'une variété linguistique. Mais c'est avant tout la typologie, en amont de toute analyse diachronique, qui permet de mettre en évidence un phénomène, ou, plus justement, l'absence d'un phénomène, par rapport aux langues apparentées ou non apparentées.

1. Cette unité morphologique peut être nuancée, notamment en ce qui concerne la morphologie verbale. 


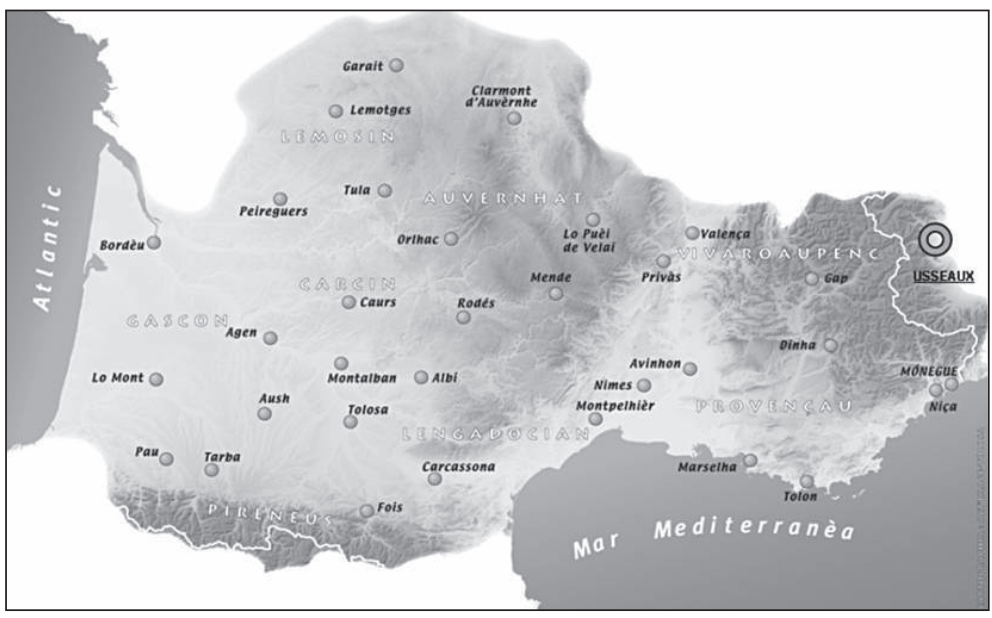

Carte 1. - Usseaux, commune occitane (Institut d'études occitanes, 2012).

\section{Cadre de l'analyse et présentation des données}

\subsection{Phénomène analysé}

Nous avons constaté à Usseaux et dans le Val Cluson un phénomène atypique en occitan moderne, et rare dans les langues romanes : l'absence du L intervocalique latin ${ }^{2}$, qui est constante sur une vingtaine d'exemples provenant de plusieurs locuteurs natifs. Ce phénomène est également attesté en portugais (Kolovrat, $1923: 225$ ) et dans une partie des variétés de la Ligurie orientale et de la Vénétie. Lorenzo Tomasin (2010) parle pour le vénitien de Lévanescent («elle evanescente») qu'il décrit comme une «approximante dorsopalatale relâchée». Georges de Kolovrat (1923), quant à lui, s'est intéressé au phénomène de vocalisation de la consonne $/ 1 /$, et écrit que :

2. Selon la norme de rédaction en usage en linguistique romane, les étymons latins ou phonèmes issus d'étymons latins seront rédigés en lettres capitales (ex : L intervocalique latin). Les phones seront présentés entres barres obliques (ex : la consonne /1/), et les phonèmes entre crochets (ex : L intervocalique latin est devenu [1] ou [r]). Dans les citations, nous reproduirons toujours le style utilisé par l'auteur, même si celui-ci est différent de celui décrit ci-dessus. 
La vocalisation de la consonne $l$ a lieu dans presque toutes les langues romanes, quoique souvent dans différentes positions phonétiques. En effet, nous l'avons observée en français, en provençal, en espagnol, en portugais, dans des dialectes italiens (ligurien, piémontais, dialectes méridionaux, sicilien), en rhétoroman de l'Ouest et partiellement en rhétoroman du Centre. (Kolovrat, 1923 : 283)

Nous avons analysé l'absence de L intervocalique en occitan alpin d'Usseaux, afin de déterminer s'il s'agit de vocalisation, d'un état résultant d'une vocalisation, ou d'un autre phénomène.

\subsection{Méthodologie}

Nous présentons ici un tableau de ces résultats (Tableau 1). Ces données ont été recueillies par élicitation à partir d'étymons latins élicités en français. Les résultats présentés dans le tableau ci-dessous ont été obtenus auprès de trois locuteurs : un homme et une femme d'une soixantaine d'années et un homme de 84 ans. Ils sont classés selon la voyelle précédant le $\mathrm{L}$ intervocalique et par contexte phonologique. Nous présentons ici une transcription en Alphabet phonétique international (API) de ces résultats.

Il faut préciser qu'il s'agit de l'analyse d'une langue en danger $(\mathrm{LED})^{3}$, qui n'est plus parlée que par quelques locuteurs âgés (une quinzaine au total pour la commune d'Usseaux). Cet échantillon peut donc paraitre minimal, mais il constitue cependant un échantillon représentatif de la population de locuteurs actifs et natifs de la commune.

3. L'occitan vivaro-alpin est considéré comme «en danger» selon des analyses «quantitatives» à l'origine du Livre Rouge (UNESCO : 1983), du site de l'Ethnologue (SIL : 1990) et de l'Atlas des Langues en danger (UNESCO : 2008). De plus, notre analyse «qualitative» sur le terrain, comme elle est pratiquée par les membres de l'axe LED-TDR (Langues En Danger : Terrain, Documentation, Revitalisation) du Laboratoire Dynamique du Langage de l'Université Lyon 2, nous a permis de confirmer cette situation pour la variété d'occitan parlée dans la commune d'Usseaux. 


\begin{tabular}{|c|c|c|c|c|c|}
\hline \multirow[t]{2}{*}{ V } & \multirow{2}{*}{$\begin{array}{l}\text { Cont } \\
\text { extes }\end{array}$} & \multirow{2}{*}{$\begin{array}{c}\text { Etymons } \\
\text { latins }\end{array}$} & \multicolumn{3}{|c|}{ Occitan alpin d'Usseaux } \\
\hline & & & $\begin{array}{l}\mathrm{W} \\
\text { (homme, } \\
60 \text { ans) }\end{array}$ & $\begin{array}{l}M \\
\text { (femme, } 64 \\
\text { ans) }\end{array}$ & $\begin{array}{l}\text { J } \\
\text { (homme, } \\
84 \text { ans) }\end{array}$ \\
\hline \multirow{6}{*}{ A } & \multirow[b]{2}{*}{$\mathrm{aLa}$} & PALA & /pool & /poo/ & l'poal \\
\hline & & ALA & fool & /'oal & /'oa/ \\
\hline & aLe & $\begin{array}{l}\text { CALENDA } \\
\mathrm{S}\end{array}$ & /t'saenda/ & /t'saenda/ & /t'saenda/ \\
\hline & aLi & GALINA & /dza'ma/ & /dza'mo/ & /dza'ma/ \\
\hline & \multirow[b]{2}{*}{ aLo } & CALORE & /t'saur/ & /t'saur/ & /t'saur/ \\
\hline & & *TALONE & /taõ/ & /'taô/ & /'taõ/ \\
\hline \multirow[b]{2}{*}{$\mathrm{E}$} & \multirow[b]{2}{*}{ eLa } & *STELA & /e'tea/ & le'tea/ & /e'tea/ \\
\hline & & CANDELA & /tsâ'dea/ & /tsã dea/ & /tsẫdea/ \\
\hline I & iLa & PILARE & /'peo/ & /'puo/ & I'pəo/ \\
\hline \multirow[b]{2}{*}{$\mathrm{O}$} & oLo & COLORE & f'kuur/ & /'kuur/ & /'kuur/ \\
\hline & oLu & $\begin{array}{l}\text { VOLUNTA } \\
\text { TE }\end{array}$ & $\mathrm{NE}^{3}$ & /'vuunta/ & /'vuunta/ \\
\hline \multirow[t]{3}{*}{$\mathrm{U}$} & oLa & $\begin{array}{l}\text { MOLA } \\
+ \text { RE }\end{array}$ & f'emuol & /'emuo/ & l'emuol \\
\hline & \multirow[b]{2}{*}{ uLa } & MULA & I'mjoel & I'mjool & I'mjos/ \\
\hline & & TABULA & /tawa/ & /'tawa/ & /'tawa/ \\
\hline
\end{tabular}

Tableau 1. - Évolution du L simple intervocalique latin en occitan alpin d'Usseaux.

\subsection{Apport de la typologie}

Nous pouvons proposer plusieurs hypothèses pour expliquer l'absence de L intervocalique dans nos données. Le phénomène pourrait être lié à un rhotacisme (1). Ce phénomène a déjà été observé dans les langues romanes, même s'il ne concerne alors pas la consonne /1/ mais plutôt les consonnes /s/ ou /z/. Il pourrait également s'agir d'un processus de vocalisation du L intervocalique (2) - la vocalisation du L se rencontrant ailleurs dans les langues romanes, ainsi que dans d'autres langues du monde (comme en anglais) mais le plus souvent en position finale. Et enfin, il pourrait s'agir d'une chute du L simple intervocalique latin (3), ce qui est rare en occitan et dans les langues romanes - et, plus généralement, dans les langues du monde - mais néanmoins attesté en 
portugais et dans quelques variétés de Ligurie et de Vénétie comme mentionné précédemment.

(1) Phénomène lié au rhotacisme hérité du substrat ligure : $-\mathrm{L}->/ \mathrm{r} /$

(2) Vocalisation du L intervocalique : $-\mathrm{L}->/ \mathrm{w} /$

(3) Chute du L intervocalique : $-\mathrm{L}->/ \varnothing /$

Nous verrons dans un premier temps quelques cas d'évolution de $\mathrm{L}$ simple intervocalique dans les langues romanes, et nous expliquerons ensuite ce phénomène pour la commune occitane d'Usseaux dans une perspective diachronique à long terme et à court terme.

\section{Approche typologique de l'évolution du $L$ intervocalique latin dans les langues romanes et en occitan}

\section{1. Évolution des liquides intervocaliques latines dans les langues romanes}

Jacques Allières a écrit, concernant les consonnes simples et les consonnes géminées (intervocaliques) des langues romanes que «l'ouest innove tandis que l'est se montre conservateur» (Allières, $2001: 30$ ). Selon lui, l'opposition simple $v s$ géminée conservée à l'est se transpose à l'ouest où «la géminée se simplifie et la simple s'amuït» (Allières, 2001 : 32). À Usseaux, qui est pourtant un parler roman central, nous verrons que les caractéristiques de l'ouest s'appliquent.

Les liquides intervocaliques latines ont, de manière très générale, été maintenues dans les langues romanes. Le cas du L intervocalique est un peu à part, en ce sens qu'il a parfois subi une rhotacisation, puis a été effacé. Selon Édouard Bourciez (1967 : 168-169), les liquides latines ont persisté dans l'ensemble du domaine roman dans la période primitive. Ce n'est que plus tard que des changements sont intervenus, notamment en portugais et à l'est du domaine en ce qui concerne le rhotacisme. /l/ y est passé à /r/ car la pointe de la langue entre en vibration. Ces changements ont été attestés à l'ouest de la Haute Italie, en Ligurie, en Lombardie, et sur quelques points du Piémont et de l'Émilie, le /r/ issu de /1/ ayant disparu par la suite. 
On voit bien ici que la tendance du L intervocalique à devenir $/ \mathrm{r} /$ est une réalité; même si, en règle générale, les liquides latines ont plutôt persisté. Dans le cas du rhotacisme, et notamment en Italie, le /r/ issu du L a disparu par la suite.

Gerhard Rohlfs, dans sa recherche sur le TLIO (Tesoro della lingua italiana delle origini), a constaté la chute complète de $\mathrm{L}$ en position finale devant /i/ dans certains dialectes septentrionaux : dans l'ancien émilien, l'ancien lombard, et l'ancien piémontais, avec un passage par un stade intermédiaire [ł] qu'il décrit comme une latérale palatale.

La caduta completa di $l$ davanti ad $i$ (attraverso uno stadio intermedio $t$ [cioè : latérale palatale,, ] si verifica anche nell'antico emiliano (tai, mai, bavii, quai, faxoi), nell antico lombardo (quai, angey 'angeli'), nell'antico piemontese (figloy, quay), cosicché risulta giustificata la domanda se le forme toscane [...] non riconducano ad influssi di provenienza settentrionale. (Rohlfs, $1996: 221$ )

Voyons maintenant quelques autres exemples d'évolution de L simple intervocalique latin dans les langues romanes.

\subsection{Conservation}

Le cas le plus fréquent est le cas de conservation, que l'on observe, entre autres, en français, italien, espagnol et occitan provençal.

(4) ALA > aile (fr.), ala (it.), ala (esp.), alo (prov.)

(5) CALORE > chaleur (fr.), calore (it.), calor (esp.), calour (prov.)

(6) TALONE > talon (fr.), tallone (it.), talòn (esp.), taloun (prov.)

\subsection{Rhotacisme}

Le second cas d'évolution possible du L intervocalique est le rhotacisme. On l'observe notamment dans certaines variétés d'occitan alpin, et aussi en provençal central et maritime. Selon Jean-Claude Bouvier (1976: 17) : «En position intervocalique, $l$ est devenu $r$ dans toute une partie de la Drôme.» Il cite les exemples de ALA et *STELA, comme en (7) et (8).

(7) ALA > ara/ala

(8) *STELA > èstyaro/étèlo 
Dans les autres langues du monde, les cas de rhotacisme $/ 1 />/ \mathrm{r} /$ ne sont pas attestés, et cela semble être réservé aux langues romanes et en particulier aux dialectes italiques. Cependant, d'autres formes de rhotacisme sont attestées, notamment concernant les consonnes $/ \mathrm{z} /$ et $/ \mathrm{s} /$. C'est le cas des langues germaniques à l'exception du gotique. Antoine Meillet le décrit ainsi :

$*_{s}$ intervocalique a tendu à passer à $*_{z}$, dans les mêmes conditions où $* f, p, x$ se sont sonorisés en $b^{h}, d^{h}, \gamma$ et $* z$, qui s'est maintenu en gotique, a passé à un phénomène de type $r$ en nordique et en germanique occidental. (Meillet, $1922: 47$ )

En ce qui concerne les autres langues indo-européennes, le grec est la seule langue concernée par le rhotacisme. Certains dialectes de grec ancien ont vu leurs /s/ intervocaliques passer à $/ \mathrm{r} /$ :

Tout donne à penser que l' $r$ du grec ancien et de l'indo-européen était, comme l'est encore celui du grec moderne, un $r$ apical [...]; c'est ce que supposent, notamment, pour le grec ancien, les faits de rhotacisme (passage dialectal de s sonorisé à $r$, soit entre voyelles [...], soit en fin de mot). (Lejeune, $1955:$ 121-122)

\subsection{Vocalisation}

Un autre cas d'évolution de L simple intervocalique latin est la vocalisation, même si Georges Straka (1968 : 387-394) décrit ce cas comme relativement rare :

Le changement de $1^{\prime} l$ intervocalique en $z^{4}$ (ou en $u^{5}$ ) n'est attesté que rarement, dans quelques patois languedociens, francoprovençaux et portugais. [...] L' $l$ intervocalique a abouti à la consonne bilabiale $w$ qu'on peut actuellement observer chez de nombreux Polonais [...] ou dans le domaine roman, à divers points du Massif Central [...] Cependant, en roman, ce $w$ a le plus souvent évolué soit vers une labio-dentale, soit - dans le cas où l'affaiblissement s'est poursuivi - vers une disparition complète.

On notera que Georges Straka (1968 : 387-394) fait mention d'une possible «disparition complète » du phonème, sans pour autant citer d'exemples.

4. Consonne fricative alvéolaire.

5. Voyelle postérieure. 
Dans les langues du monde, la vocalisation du L intervocalique n'est pas attestée ailleurs que dans les langues romanes. Elle existe cependant dans d'autres contextes, notamment en position finale. On peut notamment citer le cas de l'anglais :

The vocalisation of dark /1/ in syllable rhymes is widespread in the varieties of English spoken in the South Eastern part of Britain (Bowyer, 1973; Hardcastle and Barry, 1989; Hudson and Holloway, 1977; Meuter, 2002; Przedlacka, 2001; Spero, 1996; Tollfree, 1999; Trudgill, 1986; Wells, 1982) (indeed, it appears to be categorical in some varieties there) and extends to many other dialects including American English (Ash, 1982; Hubbell, 1950; Pederson, 2001), Australian English (Borowsky, 2001; Borowsky and Horvath, 1997; Horvath and Horvath, 1997, 2001, 2002), New Zealand English (Bauer, 1986, 1994; Horvath and Horvath, 2002) and Falkland Island English (Sudbury, 2001). (Johnson \& Britain, 2007 : 295)

\subsection{Chute}

Les cas attestés de chute de L intervocaliques sont rares dans les langues romanes, mais on peut cependant observer le phénomène dans certains dialectes d'Italie septentrionale comme le Vénétien. Lorenzo Tomasin rapporte l'existence d'un $L$ évanescent, en position initiale ou intervocalique, avec ou sans contact avec une voyelle palatale. Dans le cas d'absence de contact avec une voyelle palatale, il décrit ce L comme [e $]$ ]; en cas de contact avec une voyelle palatale, il semble disparaitre totalement :

Nel dialetto veneziano di oggi il fonema /// ha tre varianti allofoniche contestualmente condizionate. [...] b. Un'altra [e] , che si realizza in posizione iniziale o intervocalica, purché non a contatto (nemmeno in fonosintassi) con vocale palatale, comunemente chiamata elle evanescente e più puntualmente descritta, in termini articolatori, come «approssimante dorsopalatale rilassata (ess.: gondo[e ]a 'gondola', ba[e] ]ar 'ballare', ba[e ]on 'pallone', so[e ]o 'solo', par[e]ori 'per loro', 'non no [e ] a vogio la voglio', c. infine una terza, nelle stesse posizioni di (b) ma a contatto - anche in fonosintassi - con una vocale palatale, corrispondente al dileguo (Ø) (ess.: fiàr 'filare', vea 'vela', de ana 'di lana', da egno 'da legno'). (Tomasin, 2012 : 729) 


\subsection{Autres cas}

On notera enfin le cas intéressant de l'auvergnat, qui est, semble-t-il, le plus créatif en matière de traitement de L intervocalique, puisqu'il peut se maintenir, se vocaliser, se rhotaciser ou devenir $v$ ou $g$. Pierre Nauton explique ce phénomène comme suit :

Dans le domaine gallo-roman, c'est le Massif Central qui présente aujourd'hui les aboutissements les plus variés: /l/, /w/, /v/, /g/, /r/. (Nauton, $1974: 1$ )

\section{L'auteur propose une première explication à ce phénomène :}

La cause première de l'évolution [de -L-] est un affaiblissement, dû à la position intervocalique du phénomène et comparable à celui des occlusives dans la même position. [...] Dans bien des cas, l'évolution s'est poursuivie par la détente de la pointe de la langue qui s'est abaissée derrière les incisives inférieures. Le dos de la langue se trouvait alors dans la position des phénomènes postérieurs $o, u$ ou $w$, qui se sont dégagés tout naturellement. [...] L'articulation $w$, menacée de disparition, s'est renforcée en $v$ ou $g$.» (Nauton, 1974 : 190-192).

Les phénomènes de rhotacisation et de vocalisation, loin d'être opposés, auraient donc selon l'auteur la même cause : un affaiblissement articulatoire qui provoque, dans un cas, l'abaissement total de la langue, et dans l'autre, une simple détente de la pointe de la langue. On notera que lui aussi mentionne une «menace de disparition».

\subsection{Synthèse}

Cette comparaison avec les langues romanes et les autres variétés d'occitan nous permet de mettre en évidence de grandes tendances d'évolution d'un phénomène phonologique : l'évolution du L intervocalique latin. La typologie nous permet, non seulement de constater la présence de ce phénomène linguistique intéressant, mais aussi de l'expliquer en formant des hypothèses sur la base de l'évolution de ce phénomène dans d'autres langues ou dans d'autres variétés de la même langue. On constate que l'affaiblissement de L en position intervocalique n'est pas attesté ailleurs que dans les langues romanes, ce qui nous permet de dire que ce phénomène est propre à l'évolution du L intervocalique latin, et de dégager un trait d'évolution caractéristique des langues romanes. Les phénomènes de vocalisation et de rhotacisme semblent liés, et on note quelques cas de chute de L notamment dans des dialectes d'Italie septentrionale. Il nous faudra donc analyser nos résultats plus en détails 
afin de situer le cas de la commune d'Usseaux dans ces divers cas d'évolution. Une perspective diachronique à long terme constitue un point de départ intéressant pour éclaircir cette question.

\section{3. Évolution du L intervocalique latin en occitan alpin : perspectives diachroniques}

\subsection{Perspective diachronique à long terme}

Parmi les descriptions diachroniques des parlers voisins, l'ouvrage de Jean Sibille sur les parlers du Briançonnais à partir de textes religieux du Moyen Âge s'étend sur le cas du L intervocalique :

Dans une partie des parlers alpins et provençaux actuels, le continuateur de L latin intervocalique passe à $/ \mathrm{f} /{ }^{6}$, se confondant ainsi avec le continuateur de R intervocalique; tandis que LL intervocalique aboutit à $/ 1 /$. Cette situation suppose une étape antérieure dans laquelle on a deux types de $l$, sans que pour autant $l$ issu de L soit encore confondu avec $/ \mathrm{r} /$. Ce $l$ issu de L est encore attesté dans les parlers particulièrement conservateurs de Pragela (Haut-Cluson). [...] Il a souvent été qualifié de 'vélaire'; d'après la description qu'en fait Alberto Talmon (p. 16), peut-être faudrait-il plutôt le qualifier d'“apico-palatal rétroflexe vélarisé' (nous proposons la notation $\left[\mathrm{l}^{\mathrm{Y}}\right]$ plutôt que [ł]). (Sibille, 2007 : 476)

Pour résumer, cette corrélation des liquides établie par Jean Sibille (d'après les données d'Alberto Talmon (1914) en ce qui concerne le Haut-Cluson ${ }^{7}$ ) confirme ce que nous avons vu précédemment concernant l'évolution du L simple intervocalique dans les langues romanes (cf. Tableau 2). Si l'on s'intéresse maintenant uniquement à la première ligne (Haut-Cluson), on constate que ces résultats correspondent à ce que nous avons pu constater à Usseaux lors d'un terrain en 2010, au sujet de l'évolution de LI et de LL, mais pas celle de L.

6. Consonne battue.

7. Le système décrit par Alberto Talmon (1914) pour Pragela constitue un fossile de la situation médiévale. 


\begin{tabular}{|c|c|c|c|c|c|}
\hline & LI & LL & $\mathrm{L}$ & $\mathbb{R}$ & RR \\
\hline 1 & $|\mathbf{K}|$ & $1 /$ & $\mid \mathbf{y} /$ & $\mathrm{r}^{\mathrm{v} /}$ & $/ \mathrm{r} /$ \\
\hline 2 & $\langle/$ & $1 /$ & & $\%$ & $\mid \mathrm{r} /(\mathrm{R})$ \\
\hline 3 & $\| \boldsymbol{\Lambda} \mid$ & & 1/ & & $/ \mathbf{r} /$ \\
\hline 4 & $\mid \Lambda^{\prime}(\mathrm{j})$ & & /1/ & d 1 & $\mathrm{r} / \mathrm{R})$ \\
\hline 5 & $|K|$ & $1 /$ & $\mathrm{lg} / \sim / \mathrm{v} /$ & $\mid \mathrm{d} /$ & $\mathrm{r} / \mathrm{R})$ \\
\hline
\end{tabular}

Tableau 2. - Corrélation des liquides en occitan (Sibille, $2007: 477)^{8}$.

Par conséquent, pour LI (9) et LL (10) on a bien :

(9) FILIA $>$ [fiKo]

(10) BELLU > [bel].

Par contre, pour L simple, nous obtenons des résultats sensiblement différents, comme évoqué précédemment. On peut donc avancer que cette étape du L apico-palatal rétroflexe vélarisé est à considérer, mais que le L intervocalique - en tout cas pour cette localité du Haut-Cluson a subi une évolution postérieure, par simplification articulatoire.

\subsection{Perspective diachronique à court terme}

Si l'on s'intéresse maintenant à une perspective diachronique à court terme, on trouve dans des ouvrages sur des parlers voisins, ainsi que dans des écrits locaux des années 1960, un $<\nmid>9$ qui est défini comme une laryngale et qui se rapproche donc du /1/ vélaire évoqué par Jean Sibille. Nous avons donc pensé qu'il serait intéressant de regarder de plus près nos exemples pour analyser la présence d'une trace éventuelle de ce $l$.

8. Légende :

1. Haut-Cluson (Pragela)

2. Escartons de Briançon et Oulx

3. Val Germanasca et Moyen-Cluson

4. Occitan méridional

5. Gévaudan et Haute-Auvergne.

9. Ce graphème a été constaté dans plusieurs écrits. Il est décrit comme représentant un /1/ laryngal. 


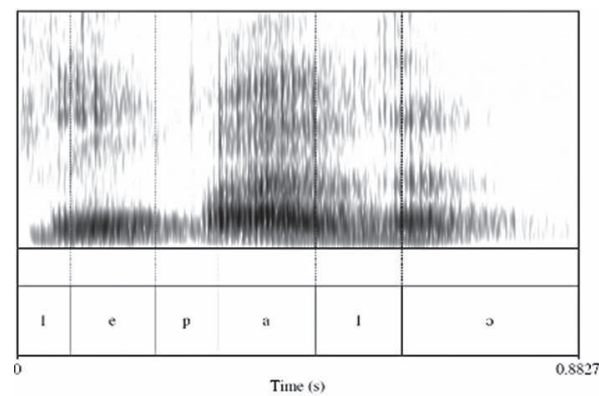

Spectrogramme $1:$ :l'épaule».

Le Spectrogramme 1 montre un mot contenant un /1/, «épaule» ([e'palə] < spalla < spatla < spatula). Les calculs réalisés sur le logiciel Praat nous montrent bien une constriction entre les deux voyelles finales, qui témoigne de la présence d'une consonne latérale.

En revanche, si l'on regarde maintenant le mot «pelle» (Spectrogramme 2) (< pala) de notre liste, on constate qu'il n'y a aucune constriction entre les deux voyelles.

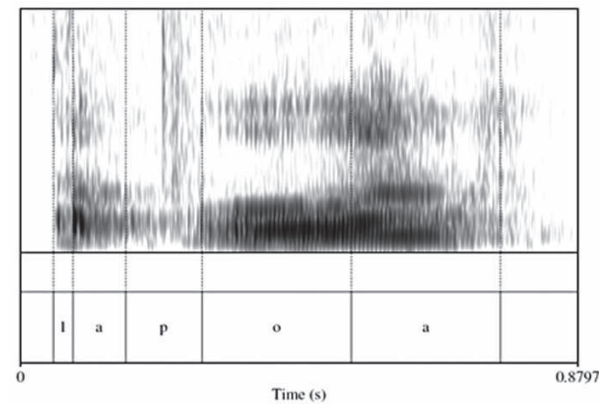

Spectrogramme $2:$ «la pelle».

On constate donc la disparition totale du L intervocalique latin en occitan alpin d'Usseaux. Cette évolution s'est produite au cours du $\mathrm{xx}^{\mathrm{e}}$ siècle très certainement à partir de la situation décrite par Alberto Talmon (1914).

\section{Conclusion}

Dans sa contribution à la description et à l'histoire des consonnes L, Georges Straka explique que : 
Les traitements de l' $l$ dans les trois positions examinées ont indiscutablement un même caractère. Qu'ils aient abouti à $w, r$ apical, $r$ pharyngal ou à zéro, ils ont été provoqués par une même cause initiale et cette cause réside sans le moindre doute dans un affaiblissement articulatoire : dans le relâchement de l'l intervocalique dû à l'aperture des voyelles voisines [...]. La variété des résultats issus de cette impulsion initiale s'explique par des variations d'énergie ou, plutôt, de faiblesse articulatoire non seulement selon les trois positions, mais aussi selon les époques, les lieux et les groupes sociaux parlant les idiomes en question. (Straka, $1968: 410$ )

À Usseaux, le L intervocalique latin a donc évolué vers une disparition, due à un affaiblissement articulatoire supposant une étape antérieure de vocalisation, et passant par une étape initiale de vélarisation.

L'étude de ce phénomène en occitan alpin nous donne un bon aperçu de ce que la typologie peut apporter à la linguistique descriptive. Cela nous aura permis de mettre en évidence l'absence du L intervocalique latin, mais aussi d'expliquer cette absence, grâce à ce que l'on sait de l'évolution de L dans d'autres langues. Une approche diachronique s'est également avérée nécessaire pour l'explication de ces phénomènes. Nos recherches ultérieures concernant le parler alpin d'Usseaux se porteront sur l'évolution de /1/ final et implosif, ainsi que sur la consonne /r/, afin de nous permettre d'avoir un aperçu du comportement des liquides de cette langue dans différents contextes.

\section{RÉFÉRENCES BIBLIOGRAPHIQUES}

Amaro L. (2008) : Étude sur le parler occitan alpin d'Usseaux, phonologie et expression de l'espace, mémoire de M2, Université Lumière-Lyon 2. Allières J. (2001) : Manuel de linguistique romane, Honoré Champion, Paris.

Bourciez E. (1967) : Éléments de linguistique romane, Éditions Klincksieck, Paris.

Bouvier J.-C. (1976) : Les parlers provençaux de la Drôme. Étude de géographie phonétique, Paris, Librairie C. Klincksieck.

JoHnson W. et BRITAIN D. (2007) : «L-vocalisation as a natural phenomenon: explorations in sociophonology », Language Sciences, 29, 294315.

Kolovrat G. de (1923) : Étude sur la vocalisation de la consonne l dans les langues romanes, Paris, Jouve \& $\mathrm{C}^{\mathrm{ie}}$.

LeJeune M. (1955) : Traité de phonétique grecque, Paris, C. Klincksieck. 
Meillet A. (1922) : Caractères généraux des langues germaniques, Paris, Hachette.

Nauton P. (1974) : Géographie phonétique de la Haute-Loire, Institut de linguistique romane de Lyon, 29.

Rohlfs G. (1966) : Grammatica storica della lingua italiana e dei suoi dialetti: fonetica, Turin, Einaudi.

SibILle J. (2007) : La passion de saint André. Édition critique suivie d'une étude linguistique comparée, Paris, Honoré Champion.

SibILle J. (2012) : «Parentés génétiques, affinités aréales et évolutions spécifiques dans les parlers occitans des vallées d'Oulx et du Haut-Cluson (Italie)», Études de linguistique gallo-romane, Presses universitaires de Vincennes, 67-82.

STRAKA G. (1968) : «Contribution à la description et à 1'histoire des consonnes L», Travaux de linguistique et de littérature, 6/1, 267-326.

Talmon A. (1914) : Saggio sul dialetto di Pragelato, Archivo glottologico italiano XVII, 1-101.

Telmon T. (2008) : «Osservazioni fonetiche», dans P. Scheuermeier, Il Piemonte dei contadini, 1921-1932, 2, 364-381.

Tomasin L. (2010) : «La cossidetta "elle evanescente" del veneziano: fra dialettologia e storia linguistica», dans G. Ruffino et M. D’Agostino (dir.), Storia della lingua italiana e dialettologia, Palermo, Centro di studi filogici e linguistici siciliani, 729-751.

Sitographie (dernière consultation : juin 2012)

ETHNOLOGUe (L') : <www.ethnologue.com>.

IEO (Institut d'Études Occitanes) : <www.ieo-oc.org >.

UNESCO, Atlas des langues en danger: <www.unesco.org/culture/ languages-atlas/fr/atlasmap.html>.

UNESCO Red Book on Endangered languages in Europe : <www.helsinki. fi/ tasalmin/europe_index.html>. 